\title{
ULTRASOUND METHOD EVALUATION OF LOWER UTERINE SEGMENT IN PATIENTS WITH PREVIOUS UTERINE CAESAREAN SECTION
}

\author{
Langrate Anna ${ }^{1,2}$, Krastina Zane $^{1,2}$, Vītina Sandra ${ }^{1}$
}

${ }^{1}$ Maternity care center, Pauls Stradins Clinical University Hospital, Riga, Latvia

${ }^{2}$ Faculty of medicine, University of Latvia, Riga, Latvia

\section{INTRODUCTION}

Caesarean section has reached high prevalence in population, becoming the most common surgical operation for women over the world. However, operation is associated with two important risk factors: the chance of uterus scar rupture during next pregnancy and mother's death.

Vaginal delivery is one of the options how to reduce /smooth out increasing value of C-sections. The risk of uterine rupture in the presence of an lower segment C-section scar is related directly to the degree of thinning of the lower uterine segment. Transabdominal ultrasonography (TA USG) is the most common used method to measure myometrium, from which the decision in performing C-section is made. But it is still debatable if the measurements are precise between transabdominal and transvaginal ultrasonography and between women with or without scar on the uterus. No cut-off values have been developed and tested, underlining the need for more standardized measurement techniques and nomenclature.

\section{OBJECTIVES}

The aim of prospective cohort study was to compare and to find a correlation between the measurement of lower uterine segment thickness made with transabdominal ultrasonography method and lately manually made measurements in vivo during Caesarean section.

\section{METHODS}

Research work was made in Pauls Stradiņš Clinical University Hospital, Maternity care center (Riga, Latvia) and was approved by the local research and ethics committee. Informed content was obtained from all women. During research 414 measurements of lower uterine segment (LUS) thickness were made for 69 single fetus pregnant women in 38.97 \pm 1.124 gestation week.

Based on data from anamnesis, patients were divided into 3 groups:

A group- women without a scar on uterus $(n=29)$,

B group- women with history of one performed C-section $(n=29)$,

C group- women with history of performed two or more C-sections $(n=11)$.

All patients underwent transabdominal ultrasound evaluation of the LUS by the same skilled sonographer few minutes before operation.

On ultrasound the lower uterine segment appears as a three- layered structure. We measured only the middle

layer of myometrium- the muscular layer. During C-sections measurement were made manually with sterile ruler immediately after removal of placenta-before suturation of uterus wall. Three measurements were taken during every method.

\section{RESULTS}

Table 1. Group characteristics

\begin{tabular}{|c|c|c|c|}
\hline \multirow[t]{2}{*}{ Parameter } & \multicolumn{3}{|c|}{ Results } \\
\hline & $\underset{(n=29)}{\text { A group }}$ & $\begin{array}{c}\text { B group } \\
(n=29)\end{array}$ & $\begin{array}{c}\text { C group } \\
(\mathbf{n}=11)\end{array}$ \\
\hline Age, mean \pm SD & $32.3 \pm 6.2$ & $33.72 \pm 4.5$ & $34,0 \pm 3.5$ \\
\hline Body mass $(\mathrm{kg})$, mean \pm SD & $79.8 \pm 17.8$ & $86.0 \pm 17.5$ & $78.1 \pm 11.4$ \\
\hline Heigh $(\mathrm{cm})$, mean \pm SD & $166.35 \pm 7.2$ & $169.5 \pm 4.8$ & $166.5 \pm 5.3$ \\
\hline BMI $\left(\mathbf{k g} / \mathbf{m}^{2}\right)$, mean \pm SD & $28.8 \pm 5.5$ & $30.0 \pm 6.4$ & $28.2 \pm 3.7$ \\
\hline Adiposity $\left(\mathrm{BMI} \geq 25,1 \mathrm{~kg} / \mathrm{m}^{2}\right), \mathrm{n}(\%)$ & $24(82.8)$ & $24(82.8)$ & $9(81.8)$ \\
\hline Gestation weeks, mean \pm SD, $\min . / \max$. & $39.0 \pm 0.9$ & $39.1 \pm 0.9$ & $38.3 \pm 1.7$ \\
\hline $\begin{array}{l}\text { Interval between pregnancys (years), } \\
\text { mean }\end{array}$ & $2.0[1 ; 6,5]$ & $5.0[3,0 ; 7,0]$ & $5.0[3,0 ; 6,0]$ \\
\hline Paritate, n (\%) & $29(42.0)$ & $29(42.0)$ & $11(15.94)$ \\
\hline 1 & $14(48.3)$ & 0 & 0 \\
\hline 2 & $9(31.0)$ & $22(75.9)$ & $1(9.1)$ \\
\hline 3 & $4(13.8)$ & $3(10.3)$ & $5(45.5)$ \\
\hline 4 & $1(3.4)$ & $1(3.4)$ & $2(18.2)$ \\
\hline 5 & $1(3.4)$ & $1(3.4)$ & $3(27.3)$ \\
\hline 6 & 0 & $1(3.4)$ & 0 \\
\hline
\end{tabular}

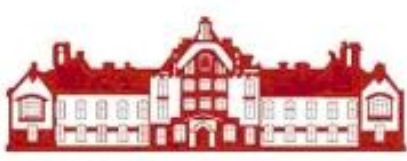

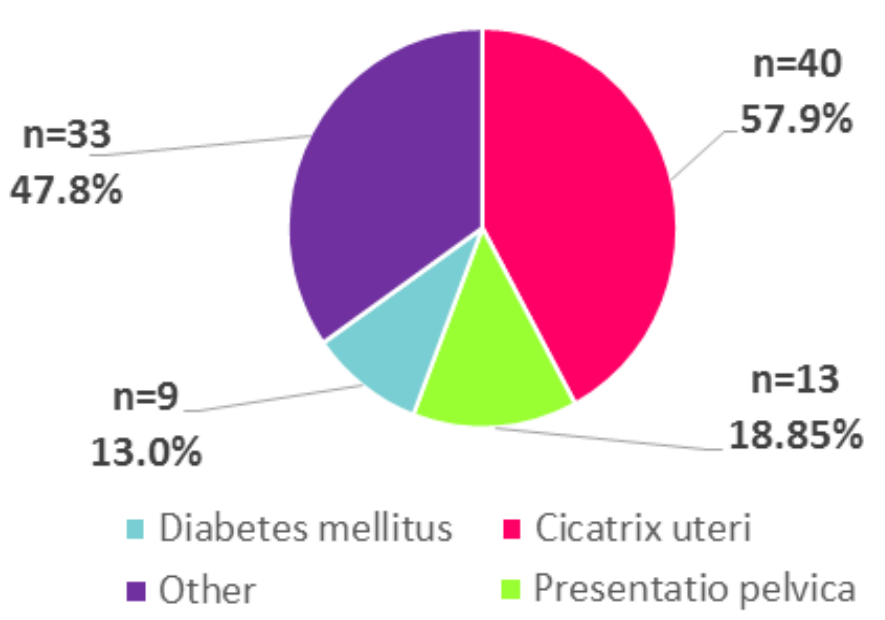

Figure 1. Most common indications for Caesarean section $(\mathrm{N}=69)$

\begin{tabular}{|c|c|c|c|c|c|c|}
\hline & \multicolumn{2}{|c|}{ Middle plane } & \multicolumn{2}{|c|}{ Lateral right plane } & \multicolumn{2}{|c|}{ Lateral left plane } \\
\hline & $\mathbf{r}_{\mathrm{s}}$ & P $<$ value* & $\mathbf{r}_{\mathrm{s}}$ & $\mathbf{P}<$ value* & $\mathbf{r}_{\mathrm{s}}$ & $\mathbf{P}<$ value* \\
\hline $\begin{array}{l}\text { A } \\
\text { group } \\
(\mathbf{N}=\mathbf{2 9})\end{array}$ & 0,540 & 0,03 & 0,238 & 0,214 & 0,225 & 0,240 \\
\hline $\begin{array}{l}\text { B } \\
\text { group } \\
(\mathbf{N}=29)\end{array}$ & 0,154 & 0,426 & 0,420 & 0,830 & $-0,073$ & $-0,707$ \\
\hline $\begin{array}{l}\text { C } \\
\text { group } \\
(\mathbf{N}=11)\end{array}$ & 0,165 & 0,628 & 0,057 & 0,869 & 0,268 & 0,426 \\
\hline
\end{tabular}

Table 2. Lower uterine segment thickness measurement correlation between available measures from ultrasonography and actual measures made in vivo.

Statistically reliable correlation between measurements from TA USG and C-section were discovered only for group A patients $(\mathrm{N}=29)$ and in only one of three measurements- in a middle plane of myometrium $(p=0,03)$. In both groups- $B$ and $C$ - there was no statistically significant correlation between the sonographic mean LUS thickness and the suraical LUS arade.

\section{0}

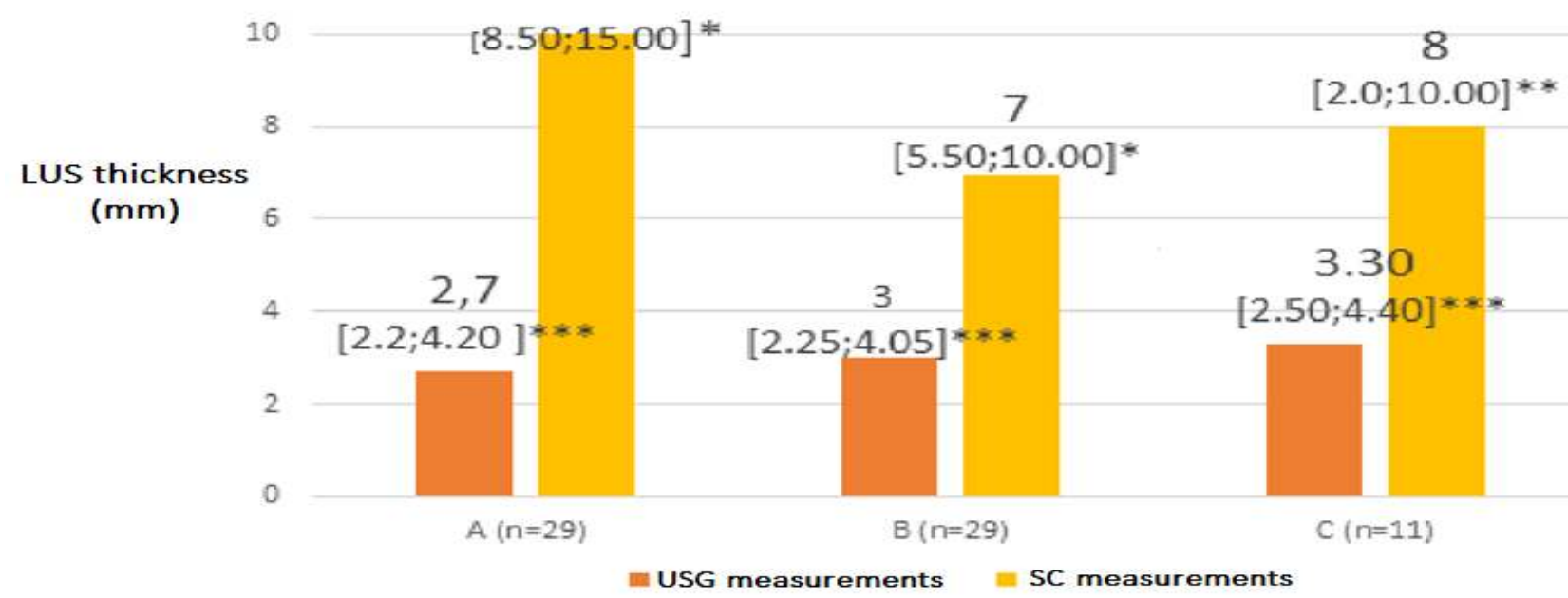

Figure 2. Image represent mean score and interquartile range in uterine wall middle plane measurements what was made with ultrasound and during C-section. ${ }^{\star}$ Mann Whitney Test with $p$ value $p<0,008,{ }^{* *}$ Kruskal Wallis tests $p<0,07,{ }^{* * *}$ Kruskal Wallis test $p<0,923$

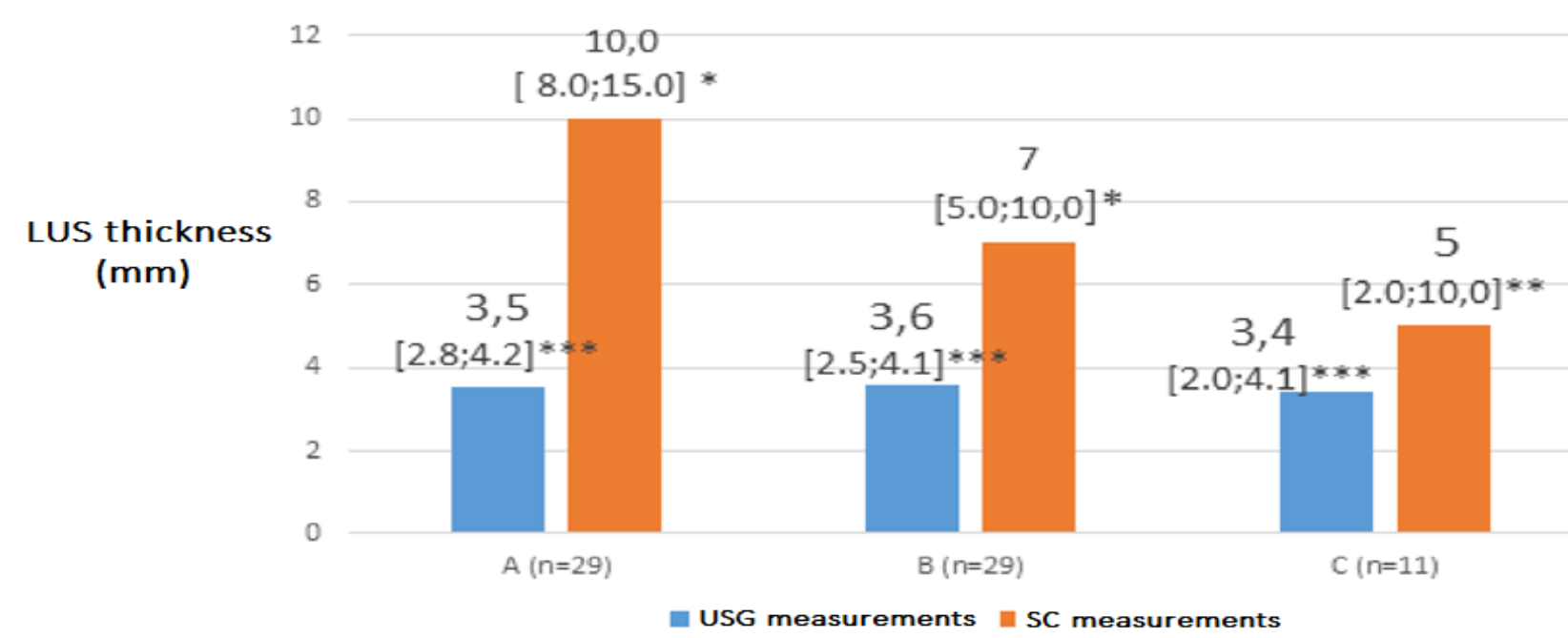

Figure 3. Image represent mean score and interquartile range in uterine wall lateral -right plane measurements what was made with ultrasound and during C-section.

${ }^{\star}$ Mann-Whitney test $p<0,001,{ }^{* *}$ Kruskal Wallis test $p<0,001$, Kruskal Wallis test $p<0,780$

CONCLUSION

- Our finding indicate that ultrasound method in third trimester is only informative for pregnant women without any scar on uterus

- Women who had not performed Caesarean section, had thicker myometrium than women who had performed Caesarean section

- Women with a scar on uterus, should consider all possible complications based on measurements not only from ultrasonography, but also must evaluate BMI (A group $p<0,097$; B group $p<0,024$; C group $p<0,020$ ), interdelivery interval $(p<0,0016)$ and patients age $(p<0,020-0,039)$, when deciding whether to perform operation

- We suggest that second trimester measurements for LUS is very importan and can predict possible rupture of uterus wall in the third trimester 